\title{
Results from a large targeted screening program for alpha-1-antitrypsin deficiency: $2003-2015$
}

Timm Greulich ${ }^{1,2^{*}}$, Christoph Nell ${ }^{1,2}$, Christian Herr ${ }^{3}$, Claus Vogelmeier ${ }^{1,2}$, Viktor Kotke ${ }^{1,2}$, Stefan Wiedmann ${ }^{1,2}$, Marion Wencker ${ }^{4}$, Robert Bals ${ }^{3}$ and Andreas Rembert Koczulla, ${ }^{1,2}$

\begin{abstract}
Background: Alpha-1-antitrypsin deficiency (AATD) is an autosomal codominant inherited disease that is significantly underdiagnosed. We have previously shown that the combination of an awareness campaign with the offer of free diagnostic testing results in the detection of a relevant number of severely deficient AATD patients. The present study provides an update on the results of our targeted screening program (German AAT laboratory, University of Marburg) covering a period from August 2003 to May 2015.
\end{abstract}

Methods: Diagnostic AATD detection test kits were offered free of charge. Dried blood samples were sent to our laboratory and used for the semiquantitative measurement of the AAT-level (nephelometry) and the detection of the S- or Z-allele (PCR). Isoelectric focusing was performed when either of the initial tests was indicative for at least one mutation. Besides, we evaluated the impact of additional screening efforts and the changes of the detection rate over time, and analysed the relevance of clinical parameters in the prediction of severe AATD.

Results: Between 2003 and 2015, 18,638 testing kits were analysed. 6919 (37.12 \%) carried at least one mutation. Of those, we identified 1835 patients with severe AATD (9.82 \% of the total test population) including 194 individuals with rare genotypes. Test initiatives offered to an unselected population resulted in a dramatically decreased detection rate. Among clinical characteristics, a history of COPD, emphysema, and bronchiectasis were significant predictors for $\mathrm{Pi}^{*} \mathrm{ZZ}$, whereas a history of asthma, cough and phlegm were predictors of not carrying the genotype $\mathrm{Pi}^{*} \mathrm{ZZ}$.

Conclusion: A targeted screening program, combining measures to increase awareness with cost-free diagnostic testing, resulted in a high rate of AATD detection. The clinical data suggest that testing should be primarily offered to patients with COPD, emphysema, and/or bronchiectasis.

Keywords: Alpha 1-antitrypsin deficiency, Asthma, Bronchiectasis, Chronic obstructive pulmonary disease, Targeted screening

\section{Background and objectives}

Alpha 1-antitrypsin (AAT) deficiency (AATD), one of the most prevalent inherited disorders in populations of European descent, is characterized by abnormally low serum levels of AAT [1]. The leading disease manifestations in AATD patients are chronic obstructive pulmonary

\footnotetext{
* Correspondence: greulich@med.uni-marburg.de

${ }^{1}$ Department of Medicine, Pulmonary and Critical Care Medicine, University

Medical Center Giessen and Marburg, Marburg, Germany

${ }^{2}$ Philipps-University Marburg, Member of the German Center for Lung

Research (DZL), Baldingerstraße, 35043 Marburg, Germany

Full list of author information is available at the end of the article
}

disease (COPD) with early onset emphysema, particularly in individuals who smoke. Further associated diseases comprise liver disease, cutaneous panniculitis, bronchiectasis, vasculitis, Wegener's granulomatosis and lung cancer [2-6].

To date, more than 100 genetic variants of the AAT gene (SERPINA1) have been identified [7]. The proteinase inhibitor (PI) MM genotype is the predominant phenotype amongst those considered as normal whereas most individuals with severe AATD are homozygous $\mathrm{PI} * \mathrm{ZZ}$ (or $\mathrm{Pi} * \mathrm{Z0}, \mathrm{Pi}^{*} 00$ ) showing a significantly reduced AAT serum concentration [8, 9]. Reports based on genetic epidemiologic surveys estimate that about 180.000 


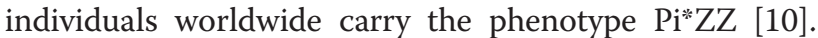
In contrast, recent data from the European Alpha-1 international registry (AIR) indicate 4.758 registered individuals with severe deficiency implying that only $3,8 \%$ of the severely affected individuals have been identified [11]. Besides, several studies have demonstrated substantial delays in the diagnosis of AATD [12, 13]. On average, AATD patients experience a diagnostic delay of 6 years and have to consult three physicians until the diagnosis is established [14]. The average age of diagnosis for AATD patients is 45.5 years, but one third is not diagnosed until their fifties [12].

ATS (American Thoracic Society)/ERS (European Respiratory Society) and WHO provided recommendations for genetic testing in order to enhance the detection of severe AATD patients $[1,15]$. Briefly, according to the WHO all individuals with COPD, emphysema or asthma or with a family history of the disorder should be screened once for AATD using a quantitative test [15]. ATS and ERS guidelines recommend testing for all patients with COPD, emphysema, or asthma with irreversible airflow obstruction [1]. Interventions undertaken to identify AATD individuals include screening and targeted detection studies [16]. Screening studies investigate unselected groups without suspicion of having AATD whereas targeted (case-finding) studies focus on individuals with a high risk for AATD. As a result, targeted approaches achieve much higher rates of detection of AATD: Several studies reported detection rate of $\mathrm{Pi}^{*} \mathrm{ZZ}$ ranging from 0.37 to $12 \%$ [17], depending on the location, the inclusion criteria and the number of individuals investigated.

In the present paper, we provide an update on the results of our targeted screening program (German AAT laboratory, University of Marburg) covering the period of August 2003 to September 2015 and including the results of 18,683 test kits.

\section{Methods}

The present study gives an update of the German targeted screening program which has been already described in detail [18]. Briefly, it combines a diagnostic service (offering a cost-free diagnostic test concerning AATD) with the aim to increase the awareness of physicians with regard to potential AATD individuals. The diagnostic approach combines the initial measurement of the AAT serum level and a targeted PCR to detect the two most common mutations ( $\mathrm{Pi}^{*} \mathrm{~S}$ and $\mathrm{Pi}^{*} \mathrm{Z}$ ) with further testing (genotyping, gene sequencing) if necessary [18].

Furthermore, "screening events" have been carried out: In these events (most often performed after a meeting of patient organizations), all individuals were invited to undergo testing, regardless of presence/absence of respiratory symptoms or a serum level. All patients gave informed consent for genetic testing. Because the study reflects a retrospective analysis of routine data, an ethics approval was not necessary.

\section{Diagnostic approach}

All samples underwent nephelometry for determination of the AAT-level and PCR for the detection of the Sand Z-allele, which was eventually followed by isoelectic focusing and gene sequencing. The results of PCR and IEF were read out and combined by two independent readers. Details regarding the laboratory methods used can be found in the online supplement (Additional file 1: Details on laboratory methods) and have been described before [18].

The results were reported to the medical doctor who had sent the testing kit. All procedures were quality controlled and based on standard operating procedures (SOPs). All laboratory procedures included appropriate negative and positive controls. The AATD Laboratory Marburg participates successfully in interlaboratory comparisons.

\section{Data storage and analysis}

The test results were entered into a Microsoft Access database. To compare detection rates between the overall results and screening events only, Fisher's exact test was used. To analyse the effect of the presence/absence of symptoms on the pre-test probability to identify $\mathrm{Pi}^{*} \mathrm{ZZ}$, a multiple logistic regression analysis was performed with the following parameters: $\mathrm{Pi}^{*} \mathrm{ZZ}$ as being the dependent variable and clinical characteristics that were recorded on the kit (acute bronchitis, asthma, bronchiectasis, COPD, dyspnoea on exertion, dyspnoea attacks, emphysema, chronic bronchitis, cough, sputum, wheezing) being the independent variables. All analyses were carried out using SPSS Version 20 (IBM, Ehningen, Germany), and GraphPad Prism Version 6 (GraphPad Software, Inc., La Jolla, USA).

\section{Results}

From August 2003 to September 2015, >50,000 test kits were sent out on request. A total number of 19,121 kits were received in our laboratory for analysis $(500-2500$ per year); 57 were not analyzable (not enough material: $n=18$; missing data: $n=39$ ) and 381 had been submitted more than once. Therefore, our final analysis includes 18,683 samples (Fig. 1).

We identified 6919 patients with at least one deficiency allele $(37.12 \%)$ including 271 individuals with rare genotypes. In descending order of frequency, we have diagnosed the following genotypes (Fig. 2): $\mathrm{Pi}$ *MM (11764; $62.97 \%), \mathrm{Pi}^{*} \mathrm{MZ}$ (4011; 21.47 \%), Pi*ZZ (1293; $6.92 \%), \mathrm{Pi}^{*} \mathrm{MS}$ (941; $\left.5.04 \%\right), \mathrm{Pi} * \mathrm{SZ}$ (348; $\left.1.86 \%\right), \mathrm{Pi}^{*} \mathrm{SS}$ $(55 ; 0.29 \%)$. In samples that underwent gene sequencing 


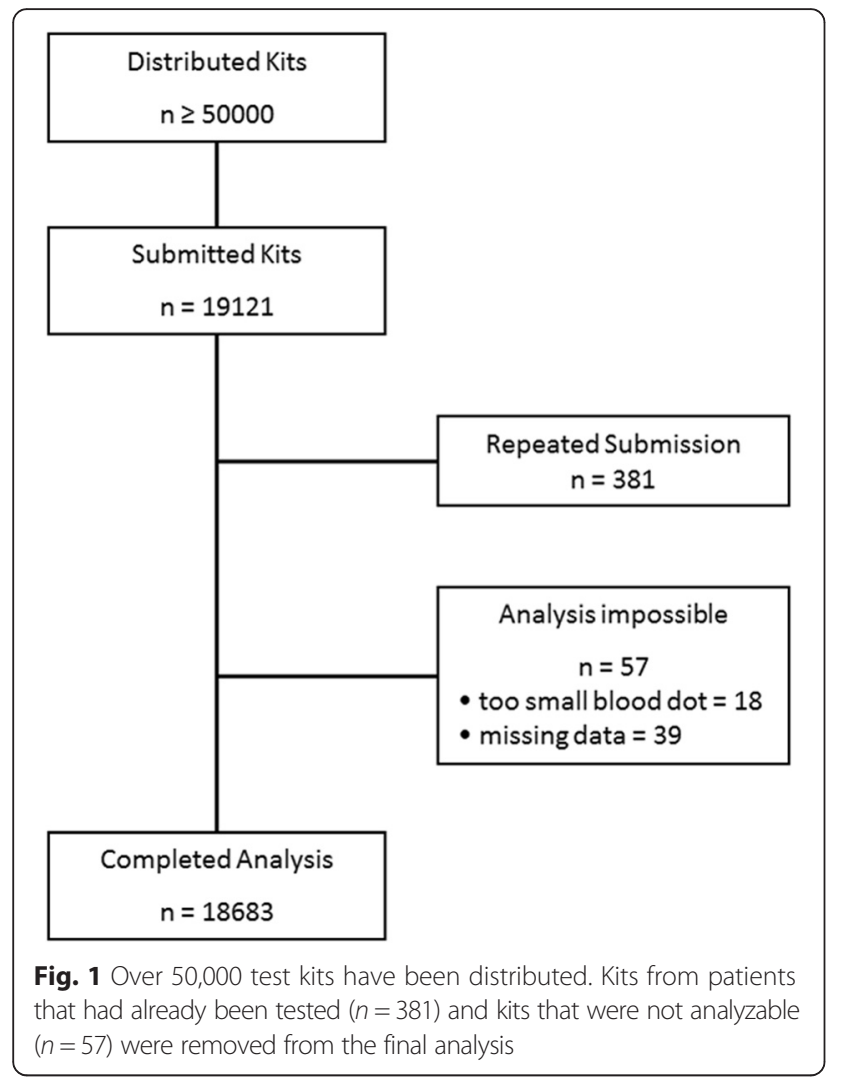

(results not clear after standard PCR and IEF), we found 271 rare genotypes.

Compared to the overall results of the laboratory, the screening events yielded significantly less positive results (at least one deficiency allele) (Table 1).

After an initial drop of the detection rate (\% samples $\mathrm{Pi}$ *ZZ in a given time period), the rate increased from 2008/09 over the following years. Exploring potential
Table 1 Results of screening events $(n=1048)$ compared to the results of the entire test population $(n=17,635)$. Displayed are percentage numbers of the laboratory population (left column) and the screening event population, respectively (right column)

\begin{tabular}{lcll}
\hline & $\begin{array}{l}\text { Laboratory } \\
\text { Population }\end{array}$ & $\begin{array}{l}\text { Screening } \\
\text { Event }\end{array}$ & $\begin{array}{l}p \text {-value } \\
\text { (Fisher-Test) }\end{array}$ \\
\hline MM & 63.48 & 88.02 & $<0.001$ \\
MS & 5.05 & 4.56 & 0.62 \\
MZ & 20.38 & 5.13 & $<0.001$ \\
SZ & 1.88 & 0.16 & $<0.001$ \\
SS & 0.31 & 0.08 & 0.25 \\
ZZ & 7.09 & 0.07 & $<0.001$ \\
Rare & 1.44 & 0 & $<0.01$ \\
\hline
\end{tabular}

reasons for that we described the three most frequent clinical characteristics that led to screening (Table 2). We found that time periods associated with high detection rates included either emphysema or COPD while the time periods with the lowest detection rates (2008/ 09 and 2010/11) did not (Table 2).

In a multivariate logistic regression analysis of clinical features of the individuals that underwent testing, we found emphysema, bronchiectasis and COPD being the strongest predictors of $\mathrm{Pi} * \mathrm{ZZ}$ (Fig. 3). Acute bronchitis, chronic bronchitis, cough, wheezing, and phlegm were associated with decreased likelihood of detecting Pi*ZZ (Fig. 3).

\section{Discussion}

From 2003 to 2015, we analyzed 18,683 DBS samples with PCR and nephelometry in our AAT diagnostic laboratory in Marburg, Germany. In 1835 (9.82 \%) of these we identified an individual with severe AATD. Our results correspond to those of other laboratories that have initiated

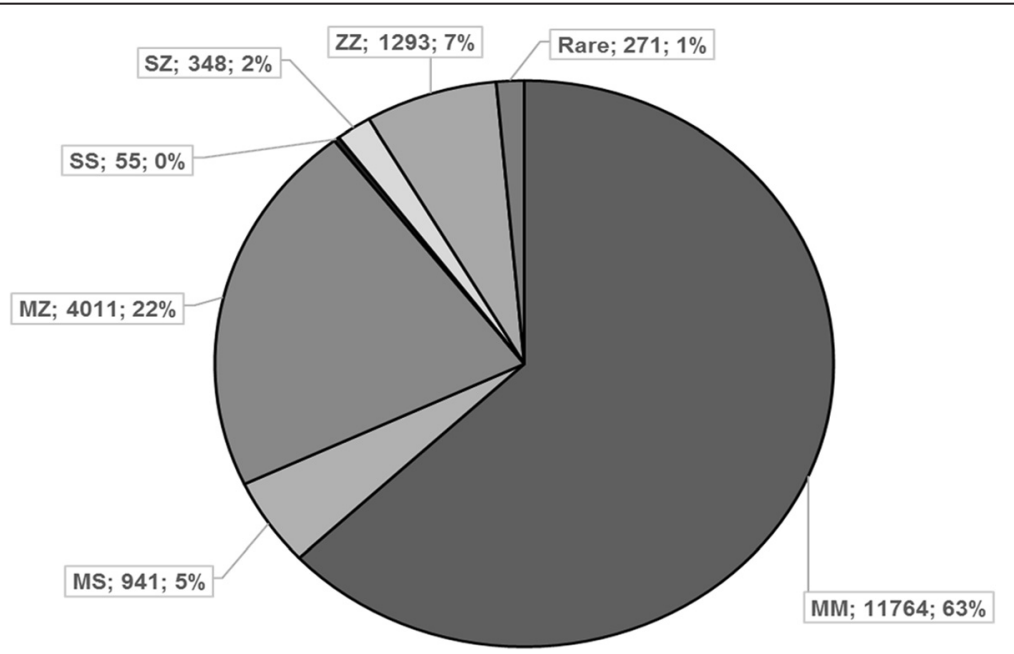

Fig. 2 Results from 18,683 kits that have been analysed successfully within the first 12 years of the laboratory (August 2003 to September 2015) 
Table 2 Displayed are the detection rates ( $\mathrm{Pi}^{*} \mathrm{ZZ}$ ) of different time periods, the characteristics of the population screened at that time (age in years; mean \pm standard deviation) and the three most frequent clinical characteristics that led to the test initiation. Numbers do not add up to 18,683 , because for some individuals the gender was not recorded and could not be deducted by the name

\begin{tabular}{|c|c|c|c|c|}
\hline & $\begin{array}{l}\text { Detection } \\
\text { rate [\%] }\end{array}$ & Age [years] & Male/female & $\begin{array}{l}\text { Most frequent clinical } \\
\text { characteristics }\end{array}$ \\
\hline \multirow[t]{3}{*}{$2003 / 04 / 05$} & 8.65 & $39.8 \pm 21.9$ & $1105 / 887$ & 1. Cough \\
\hline & & & & 2. Emphysema \\
\hline & & & & 3. Wheezing \\
\hline \multirow[t]{3}{*}{ 2006/07 } & 4.04 & $40.4 \pm 21.7$ & $1527 / 1399$ & 1. Dyspnoea on exertion \\
\hline & & & & 2. Cough \\
\hline & & & & 3. Wheezing \\
\hline \multirow[t]{3}{*}{ 2008/09 } & 4.25 & $48.9 \pm 21.6$ & 1973/1791 & 1. Dyspnoea on exertion \\
\hline & & & & 2. Cough \\
\hline & & & & 3. Phlegm \\
\hline \multirow[t]{3}{*}{ 2010/11 } & 4.98 & $48.2 \pm 20.3$ & $2127 / 1888$ & 1. Dyspnoea on exertion \\
\hline & & & & 2. Cough \\
\hline & & & & 3. Phlegm \\
\hline \multirow[t]{3}{*}{$2012 / 13$} & 7.91 & $47.9 \pm 19.1$ & 1808/1661 & 1. Dyspnoea on exertion \\
\hline & & & & 2. Cough \\
\hline & & & & 3. COPD \\
\hline \multirow[t]{3}{*}{ 2014/15 } & 11.26 & $48.5 \pm 18.8$ & $1280 / 1139$ & 1. Dyspnoea on exertion \\
\hline & & & & 2. COPD \\
\hline & & & & 3. Cough \\
\hline \multirow[t]{3}{*}{ All } & 6.85 & $45.9 \pm 20.8$ & $9820 / 8765$ & 1. Dyspnoea on exertion \\
\hline & & & & 2. Cough \\
\hline & & & & 3. $\mathrm{COPD}$ \\
\hline
\end{tabular}

targeted detection regarding AATD and focus on test populations showing clinical features of the disease.

The Italian program distributing DBS AATD tests to hospitals and clinics suggested testing of individuals with early-onset emphysema and familial cluster of

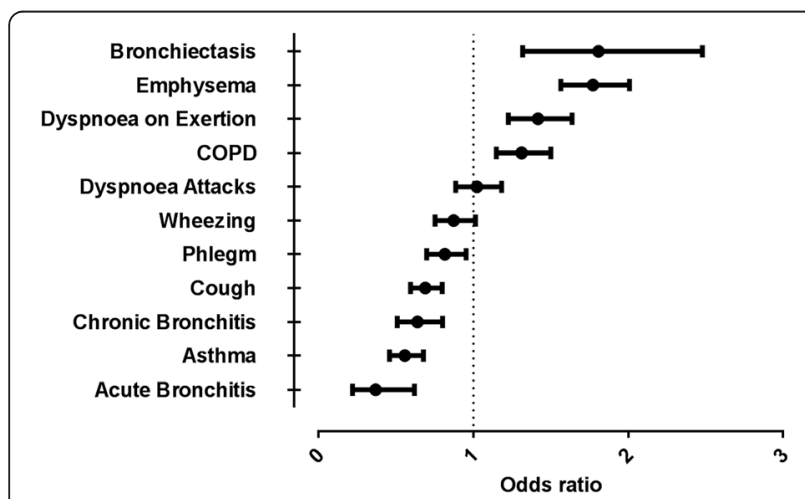

Fig. 3 Clinical characteristics predictive for the genotype Pi*ZZ (multivariate logistic regression analysis)
COPD, with a plasma AAT level $<80 \mathrm{mg} / \mathrm{dl}$ or first degree relatives of subjects with known AATD (including Pi*MZ heterozygosity) [19, 20]. Of 2127 samples, $10.6 \%$ presented with severe deficiency $\left(\mathrm{Pi}^{*} \mathrm{ZZ}, \mathrm{Pi} * \mathrm{SZ}\right.$ and rare genotypes). Carroll et al. screened 3000 individuals as part of the Irish National Targeted Detection Programme and included subjects with COPD, nonresponsive asthma, cryptogenic liver disease, first degree relatives of AATD patients and individuals with reduced serum AAT levels [21]. $3.1 \%$ were found to have a severe deficiency. Corda et al. chose an extremely selected study population displaying specific characteristics of AATD in order to perform a targeted screening study and identified $15.8 \%$ severely deficient phenotypes $\left(12 \% \mathrm{Pi}^{*} \mathrm{ZZ}\right)$ in a rather small study sample $(n=285)$ [22]. On the other hand, screening studies including non-selected patients with COPD or other respiratory clinical syndromes were not able to detect severe AATD phenotypes in more than $0.5 \%$ of the study population [23-25]. These data once more underline the importance of careful selection regarding individuals suspected to have AATD when performing a targeted screening program. To our knowledge, our analysis represents the as yet biggest targeted screening cohort in Europe $(n=18,683)$. According to estimates of the frequency of $\mathrm{Pi}^{*} \mathrm{ZZ}$ in Germany [26], 8003 individuals would be expected. Having detected 1293 individuals $(16.15 \%)$ is a higher percentage than has been reported in many other countries of the world as pointed out by a recent review [27].

Comparing our data to those previously published [18] we were able to document stability of the results over time which might derive from the continuous preselection of the study population, the high number of analyzed samples and the experience of the Marburg laboratory with applying diagnostic methods concerning AATD. While the results remained rather stable comparing the first published analysis with the recent one, it has to be acknowledged that there was a drop in the detection rate during 2008 - 2011 (Table 2). The increase after that may - at least in part - be explained by COPD becoming a more prominent cause to send samples to our laboratory, reflecting a population being more likely to suffer from severe AATD (Table 2). Regarding the relatively high detection rate in 2003 - 2005, younger patients with emphysema have been tested, again a population more likely to carry a severe deficiency SERPINA1 genotype (Table 2).

By using our test algorithm, we intended to investigate especially those patients who showed low concentrations of AAT levels in order to prevent unnecessary diagnostic steps and save costs. On the other hand, heterozygous carriers of SERPINA1 mutations may have been missed because the determination of the AAT level precedes sending of tests to our laboratory. Ferrarotti et al. (University of Pavia) perform genotyping (by PCR- 
RFLP using TaqI as the restriction enzyme) at the first stage of their algorithm simultaneously with the quantitative measurement of AAT-levels [28], resulting in a higher rate of testing in samples with normal AAT-levels. Furthermore, the testing algorithm used by the laboratory of the university in Pavia allows consecutive sequence analyses if the results of the phenotyping do not produce clear evidence about deficiency phenotypes whereas our laboratory repeats nephelometry, PCR and IEF in whole blood samples of individuals with conflicting results from DBS analyses before performing additional DNA sequencing.

We detected a relatively high number of rare genotypes other than $\mathrm{Pi}^{*} \mathrm{ZZ}$ or $\mathrm{Pi}$ "SZ among the individuals with severe AATD $(194 / 1835=10.57 \%)$ which is more than, for example, the rate of $1.7 \%$ reported by the NHLBI Registry [29], and comparable with results published by Ferrarotti et al. who recorded an $11 \%$ prevalence of subjects with severe AATD carrying rare variants [30]. In the light of a new test that identifies individuals with a Z-allele but would not detect individuals with severe deficiency without one or two $\mathrm{Z}$ alleles [31] it is reassuring to notice that only very few individuals (22 S/rare, 17 rare/ rare) would be missed by this test.

The majority of kits was returned by pulmonologists reflecting the pulmonary symptoms most patients with symptomatic AATD suffer. The low number of gastroenterologists who returned DBS to our laboratory might be explained by the fact that liver problems deriving from an underlying AATD occur directly after birth. During this phase of life testing will be usually requested by the pediatricians. Evaluating targeted AATD screening programs should also include the aspect of cost-effectiveness. In fact, it has been shown that casefinding in populations in which AATD is suspected in reasonable frequency (e.g. patients with known COPD) could satisfy cost-effectiveness criteria [32]. In line with this, our testing algorithm reveals its potential to save costs by applying phenotyping ( $<50 \%$ IEF) and sequencing $(\sim 1 \%)$ in a limited number of individuals.

With regard to clinical characteristics that would be predictive for $\mathrm{Pi}$ "ZZ we confirmed earlier reports of COPD and emphysema being important predictors. The fact that bronchiectasis was a strong predictor for $\mathrm{Pi}^{*} \mathrm{ZZ}$ is interesting, because earlier reports gave conflicting results [33-35]. Our results would support screening for AATD in this patient group that is increasingly recognized [36]. Asthma and acute bronchitis were the strongest clinical predictors of not carrying a homozygous deficiency. This confirms studies that did not report an increased prevalence of homozygous AATD in asthmatic patients [37, 38].

Concerning potential limitations of our screening program we acknowledge that targeting selected (symptomatic) individuals might result in missing asymptomatic subjects with severe AATD. This has been suggested by several studies which investigated the percentage of $\mathrm{Pi}$ *ZZ individuals with clinical symptoms. A significant proportion of homozygote $\mathrm{Pi}$ "ZZ subjects do neither develop emphysema [39] nor COPD [40]. Besides, we have only limited information about the patients, their clinical characteristics or serum AAT levels. Reporting symptoms (beyond the clinical characteristics that have been asked for), lung function, smoking habits and family screening would have been helpful in characterizing AATD individuals.

\section{Conclusions}

In conclusion, we have shown that our targeted screening program, combining an awareness campaign with cost-free diagnostic testing, resulted in a high rate of AATD detection, especially in patients with COPD, emphysema, and bronchiectasis. In the context that AATD is still an under-diagnosed disease and that recognition may entail important implications (e.g. smoking avoidance, family testing, augmentation therapy), targeted screening approaches should be continued.

\section{Ethics approval and consent to participate}

All patients gave informed consent for genetic testing. Because the study reflects a retrospective analysis of routine data, an ethics approval was not necessary.

\section{Consent for publication}

Not applicable.

\section{Availability of data and materials}

Data will not be shared. The data are subjects to further scientific analyses that may be published in the future.

\section{Additional file}

Additional file 1: Details on laboratory methods. (DOCX $12 \mathrm{~kb}$ )

\section{Abbreviations}

AATD: Alpha-1-antitrypsin deficiency; ATS: American Thoracic Society; COPD: chronic obstructive pulmonary disease; DBS: dried blood spot; DNA: deoxyribonucleic acid; ERS: European Respiratory Society; IEF: isoelectric focusing; NHLBI: National Heart, Lung and Blood Institute; PCR: polymerase chain reaction; RFLP: restriction (Enzyme) fragment length polymorphism; SOP: standard operating procedure.

\section{Competing interests}

$\mathrm{TG}, \mathrm{RB}, \mathrm{ARK}$, and $\mathrm{CV}$ have received speaking and consulting fees from Talecris Biotherapeutics (Grifols) and from CSL-Behring. MW is a former ( $>5$ years ago) employee of Talecris biotherapeutics (Grifols) and receives consulting fees from CSL-Behring.

\section{Authors' contributions}

TG, CH, VK, SW, RB, and ARK performed laboratory work. TG and CN performed the statistical analyses. TG, CH, CV, MW, RB, and ARK contributed significantly to the concept and the design of the present analysis. TG and ARK performed the first draft of the manuscript. All authors helped in revising the manuscript critically and gave their approval to the final version of the manuscript. 


\section{Acknowledgements}

We thank all employees of the alpha-1 antitrypsin laboratory at the University of Marburg for their continuous effort they put into this project.

\section{Funding}

The alpha-1 antitrypsin laboratory at the University of Marburg has been supported by Talecris Biotherapeutics (Grifols). The funder had no role in the collection, analysis, and interpretation of the data and had no role in writing the manuscript.

\section{Author details}

'Department of Medicine, Pulmonary and Critical Care Medicine, University Medical Center Giessen and Marburg, Marburg, Germany. ${ }^{2}$ Philipps-University Marburg, Member of the German Center for Lung Research (DZL), Baldingerstraße, 35043 Marburg, Germany. ${ }^{3}$ Department of Internal Medicine V, Pulmonology, Allergology, Respiratory and Environmental Medicine, Saarland University Hospital, 66421 Homburg/Saar, Germany. ${ }^{4}$ Department of Pneumology, University Hospital Essen, Ruhrlandklinik, 45239 Essen, Germany.

Received: 23 February 2016 Accepted: 13 May 2016 Published online: 10 June 2016

\section{References}

1. ATS/ERS. American Thoracic Society/European Respiratory Society statement: standards for the diagnosis and management of individuals with alpha-1 antitrypsin deficiency. Am J Respir Crit Care Med. 2003;168(7):818-900.

2. Edmonds BK, Hodge JA, Rietschel RL. Alpha 1-antitrypsin deficiencyassociated panniculitis: case report and review of the literature. Pediatr Dermatol. 1991:8(4):296-9.

3. Sveger T. Liver disease in alpha1-antitrypsin deficiency detected by screening of 200,000 infants. N Engl J Med. 1976;294(24):1316-21.

4. Lewis M, Kallenbach J, Zaltzman M, Levy H, Lurie D, Baynes R, et al. Severe deficiency of alpha 1-antitrypsin associated with cutaneous vasculitis, rapidly progressive glomerulonephritis, and colitis. Am J Med. 1985;79(4):489-94.

5. Rubinstein HM, Jaffer AM, Kudrna JC, Lertratanakul Y, Chandrasekhar AJ, Slater D, et al. Alpha1-antitrypsin deficiency with severe panniculitis. Report of two cases. Ann Intern Med. 1977;86(6):742-4.

6. Yang P, Bamlet WR, Sun Z, Ebbert JO, Aubry MC, Krowka MJ, et al. Alpha1antitrypsin and neutrophil elastase imbalance and lung cancer risk. Chest. 2005;128(1):445-52.

7. Demeo DL, Silverman EK. Alpha1-antitrypsin deficiency. 2: genetic aspects of alpha(1)-antitrypsin deficiency: phenotypes and genetic modifiers of emphysema risk. Thorax. 2004;59(3):259-64.

8. Bals R, Koczulla R, Kotke V, Andress J, Blackert K, Vogelmeier C. Identification of individuals with alpha-1-antitrypsin deficiency by a targeted screening program. Respir Med. 2007;101(8):1708-14.

9. Janciauskiene SM, Bals R, Koczulla R, Vogelmeier C, Kohnlein T, Welte T. The discovery of alpha1-antitrypsin and its role in health and disease. Respir Med. 2011;105(8):1129-39.

10. de Serres FJ, Blanco I. Prevalence of alpha1-antitrypsin deficiency alleles PI*S and $\mathrm{PI}^{*} \mathrm{Z}$ worldwide and effective screening for each of the five phenotypic classes $\mathrm{PI}^{*} \mathrm{MS}, \mathrm{PI}^{*} \mathrm{MZ}, \mathrm{PI}{ }^{*} \mathrm{SS}, \mathrm{PI}{ }^{*} \mathrm{SZ}$, and $\mathrm{PI}{ }^{*} \mathrm{ZZ}$ : a comprehensive review. Ther Adv Respir Dis. 2012;6(5):277-95.

11. Stockley RA, Dirksen A, Stolk J. Alpha-1 antitrypsin deficiency: the European experience. COPD. 2013;10 Suppl 1:50-3.

12. Campos MA, Wanner A, Zhang G, Sandhaus RA. Trends in the diagnosis of symptomatic patients with alpha1-antitrypsin deficiency between 1968 and 2003. Chest. 2005;128(3):1179-86.

13. Stoller JK, Sandhaus RA, Turino G, Dickson R, Rodgers K, Strange C. Delay in diagnosis of alpha1-antitrypsin deficiency: a continuing problem. Chest. 2005;128(4):1989-94

14. Kohnlein $T$, Janciauskiene $S$, Welte $T$. Diagnostic delay and clinical modifiers in alpha-1 antitrypsin deficiency. Ther Adv Respir Dis. 2010;4(5):279-87.

15. WHO. Alpha 1-antitrypsin deficiency: memorandum from a WHO meeting. Bull World Health Organ. 1997;75(5):397-415.

16. Aboussouan LS, Stoller JK. Detection of alpha-1 antitrypsin deficiency: a review. Respir Med. 2009;103(3):335-41.

17. Greulich T, Vogelmeier CF. Alpha-1-antitrypsin deficiency: increasing awareness and improving diagnosis. Ther Adv Respir Dis. 2016;10(1):72-84.
18. Crystal RG, Brantly ML, Hubbard RC, Curiel DT, States DJ, Holmes MD. The alpha 1-antitrypsin gene and its mutations. Clinical consequences and strategies for therapy. Chest. 1989;95:196-208.

19. Luisetti M, Massi G, Massobrio M, Guarraci P, Menchicchi FM, Beccaria M, et al. A national program for detection of alpha 1-antitrypsin deficiency in Italy. Gruppo IDA. Respir Med. 1999;93(3):169-72.

20. Ferrarotti I, Gorrini M, Scabini R, Ottaviani S, Mazzola P, Campo I, et al. Secondary outputs of alpha1-antitrypsin deficiency targeted detection programme. Respir Med. 2008;102(3):354-8.

21. Carroll TP, O'Connor CA, Floyd O, McPartlin J, Kelleher DP, O'Brien G, et al. The prevalence of alpha-1 antitrypsin deficiency in Ireland. Respir Res. 2011;12:91.

22. Corda L, Bertella E, Pini L, Pezzini A, Medicina D, Boni E, et al. Diagnostic flow chart for targeted detection of alpha1-antitrypsin deficiency. Respir Med. 2006;100(3):463-70

23. de la Roza C, Rodriguez-Frias F, Lara B, Vidal R, Jardi R, Miravitlles M. Results of a case-detection programme for alpha1-antitrypsin deficiency in COPD patients. Eur Respir J. 2005;26(4):616-22.

24. Molina J, Flor X, Garcia R, Timiraos R, Tirado-Conde G, Miravitlles M. The IDDEA project: a strategy for the detection of alpha-1 antitrypsin deficiency in COPD patients in the primary care setting. Ther Adv Respir Dis. 2011;5(4):237-43.

25. Wencker M, Marx A, Konietzko N, Schaefer B, Campbell EJ. Screening for alpha1Pi deficiency in patients with lung diseases. Eur Respir J. 2002;20(2):319-24.

26. Blanco I, de Serres FJ, Fernandez-Bustillo E, Lara B, Miravitlles M. Estimated numbers and prevalence of $\mathrm{PI}^{*} \mathrm{~S}$ and $\mathrm{PI}{ }^{*} \mathrm{Z}$ alleles of alpha1-antitrypsin deficiency in European countries. Eur Respir J. 2006;27(1):77-84.

27. Stoller JK, Brantly M. The challenge of detecting alpha-1 antitrypsin deficiency. COPD. 2013;10 Suppl 1:26-34.

28. Ferrarotti I, Scabini R, Campo I, Ottaviani S, Zorzetto M, Gorrini M, et al. Laboratory diagnosis of alpha1-antitrypsin deficiency. Transl Res. 2007;150(5):267-74.

29. McElvaney NG, Stoller JK, Buist AS, Prakash UB, Brantly ML, Schluchter MD, et al. Baseline characteristics of enrollees in the National Heart, Lung and Blood Institute Registry of alpha 1-antitrypsin deficiency. Alpha 1-Antitrypsin Deficiency Registry Study Group. Chest. 1997:111(2):394-403.

30. Ferrarotti I, Baccheschi J, Zorzetto M, Tinelli C, Corda L, Balbi B, et al. Prevalence and phenotype of subjects carrying rare variants in the Italian registry for alpha1-antitrypsin deficiency. J Med Genet. 2005;42(3):282-7.

31. Vogelmeier C, Soriano J, Janciauskiene S, Crystal RG, Ferrarotti I, Carroll T. Alpha-1-antitrypsin deficiency: honouring the past and embracing the future. Grifols' Symposium Report. Expert Rev Respir Med. 2013.

32. Shermock KM, Gildea TR, Singer M, Stoller JK. Cost-effectiveness of population screening for alpha-1 antitrypsin deficiency: a decision analysis. COPD. 2005; 2(4):411-8.

33. Cuvelier A, Muir JF, Hellot MF, Benhamou D, Martin JP, Benichou J, et al. Distribution of alpha(1)-antitrypsin alleles in patients with bronchiectasis. Chest. 2000;117(2):415-9.

34. Parr DG, Guest PG, Reynolds JH, Dowson LJ, Stockley RA. Prevalence and impact of bronchiectasis in alpha1-antitrypsin deficiency. Am J Respir Crit Care Med. 2007:176(12):1215-21.

35. Kauczor HU, Hoffmann A, Mehdikhani H, Schunk K, Schlegel J, Mildenberger $P$. Bronchiectasis and infection incidence in alpha 1-antitrypsin deficiency. The value of high-resolution computed tomography. Rofo. 1995;163(5):378-82.

36. Ringshausen FC, de Roux A, Diel R, Hohmann D, Welte T, Rademacher J. Bronchiectasis in Germany: a population-based estimation of disease prevalence. Eur Respir J. 2015;46(6):1805-7.

37. Eden E, Holbrook JT, Brantly ML, Turino GM, Wise RA. Prevalence of alpha-1 antitrypsin deficiency in poorly controlled asthma-results from the ALAACRC low-dose theophylline trial. J Asthma. 2007:44(8):605-8.

38. van Veen $\mathrm{H}$, ten Brinke $A$, van der Linden AC, Rabe KF, Bel EH. Deficient alpha-1-antitrypsin phenotypes and persistent airflow limitation in severe asthma. Respir Med. 2006;100(9):1534-9.

39. Eriksson S. A 30-year perspective on alpha 1-antitrypsin deficiency. Chest. 1996;110(6 Suppl):237S-42S.

40. Parr DG, Stoel BC, Stolk J, Stockley RA. Pattern of emphysema distribution in alpha1-antitrypsin deficiency influences lung function impairment. Am J Respir Crit Care Med. 2004;170(11):1172-8. 